\title{
Cognitive Profile of Elderly Patients with Mild Stroke
}

\author{
Arne Gramstad $^{\mathrm{a}, \mathrm{b}}$ Dag Aarsland ${ }^{\mathrm{d}}$ Halvor Naess ${ }^{\mathrm{a}, \mathrm{c}}$

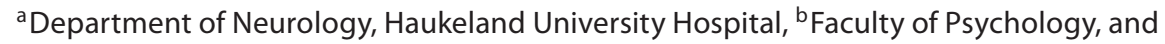 \\ 'Department of Clinical Medicine, University of Bergen, Bergen, and dPsychiatric Division, \\ Stavanger University Hospital, Stavanger, Norway
}

\section{Key Words}

Color-word interference $\cdot$ Learning $\cdot$ Memory $\cdot$ Mental state $\cdot$ Mild stroke

\begin{abstract}
Background: A pattern characterizing cognitive deficits in mild stroke could help in differential diagnosis and rehabilitation planning. Methods: Fifty patients with mild stroke (modified Rankin scale $\leq 2$ at discharge) aged $>60$ years were given the Mini Mental State Examination (MMSE), the Hopkins Verbal Learning Test-Revised (HVLT-R) and the Stroop test. Results: On HVLT-R, significant impairments were found in learning and recall, but not in delayed recall. The Stroop test revealed significant impairments in reading speed, but not in color-word interference. Using the MMSE, significant deficits were only found in the youngest age group. Conclusion: Elderly patients with mild stroke show deficits in verbal learning/recall and in reading speed, but not in the MMSE, delayed recall or color-word interference. The deficits are consistent with a mild-to-moderate brain dysfunction, with relative sparing of medial brain structures.
\end{abstract}

Copyright $\odot 2011$ S. Karger AG, Basel

\section{Introduction}

Stroke can give rise to a wide spectrum of cognitive deficits, depending upon disease variables such as size and location of the lesion, etiology and comorbidity, and background variables such as age, sex and level of functioning before disease onset. Cognitive deficits 
are important for the clinical outcome after stroke and must be taken into consideration when rehabilitation is planned [1]. Following large vascular cerebral lesions, cognitive deficits are often quite distinct and may lead to clearly visible symptoms such as aphasia, neglect and/or hemiparesis. In addition, assessment of general tasks such as language, memory, attention and executive functions is important [2]. In recent years, increasing attention has been addressed to the earliest cognitive changes in Alzheimer's disease (AD), but few studies have explored the earliest cognitive changes in patients with mild stroke, although these cognitive deficits may be of importance $[3,4]$. Among the cognitive functions studied, memory and executive functions seem to have aroused most scientific attention [5-7]. To define a cognitive profile of deficits caused by mild stroke might be helpful in the differential diagnosis between mild stroke and other causes of brain dysfunction. Differential diagnosis based on the assessment of cognitive performance may have important implications for treatment.

In patients with vascular dementia, cognitive deficits may differ from those seen in other types of dementia, e.g. $\mathrm{AD}$. In $\mathrm{AD}$, memory deficits are prominent, whereas deficits in executive function are more prominent in vascular dementia [8]. However, it is not known whether these differences occur in earlier stages, e.g. early degenerative disease or mild stroke. It has been reported that in early-stage AD medial temporal lobe structures are primarily affected [9]. These structures are critical for episodic memory, especially for delayed recall which is often impaired in early stages of AD [10]. Specific deficits in delayed recall are more prominent in AD than in vascular dementia [11]. In its earlier stages, vascular dementia tends to show deficits in executive functioning, although this is an inconsistent finding, and differential diagnosis can be difficult $[8,11]$, since executive functions, such as resistance to interference in the Stroop task, may also be affected in AD [12].

Mild stroke may or may not develop into vascular dementia. However, cognitive deficits caused by mild stroke in the elderly may in itself lead to significant problems in coping with everyday tasks and reduced quality of life. Better knowledge about typical cognitive difficulties in this group will increase the ability to plan rational rehabilitation strategies. In addition, it may be important to differentiate the cognitive profile of mild stroke from that of amnesic mild cognitive impairment (MCI). This condition often presents with neuropathological changes [13] and a memory profile similar to early $\mathrm{AD}$, and it often develops into $\mathrm{AD}$ $[14,15]$.

To investigate the cognitive profile of elderly patients with mild stroke in more detail, we administered tests of verbal learning and recall, reading speed, response inhibition and global functioning. Based on earlier observations, we expected that the Mini Mental State Examination (MMSE), a measure of global functioning, is only moderately sensitive to cognitive dysfunction in patients with mild stroke, whereas tasks such as verbal learning and recall, reading speed and interference, which are impaired in patients with mild stroke, might be more sensitive than the MMSE [16-18]. We did not, however, expect any specific deficit in delayed free recall, as seen in amnesic $\mathrm{MCI}$ and $\mathrm{AD}$.

\section{Patients and Methods}

\section{Patients}

All consecutive patients with acute stroke (the index stroke) admitted to the Stroke Unit, Department of Neurology, Haukeland University Hospital, between February 2006 and April 2008 underwent a comprehensive and detailed neurological and biomarker examination and were prospectively registered in a database (the Bergen Stroke Study). The study was approved by the local ethics committee. Fifty patients with a diagnosis of mild cerebral in- 
farction were included in the present study. Inclusion criteria were modified Rankin scale (mRS) score $\leq 2$ within 1 week of stroke onset, age $>60$ years and acute cerebral infarction on diffusion-weighted imaging (DWI). DWI was performed as part of a routine MRI protocol for stroke patients on a 1.5-tesla Siemens Magnetom (Symphony). The DWI sequence used was ep2d_diff_3scan_trace with the following specifications of parameters: field of view $230 \mathrm{~mm}$, slice thickness $5 \mathrm{~mm}$, TR 3,200 ms and TE $94 \mathrm{~ms}$. Patients with dementia and other severe medical conditions were excluded. A study nurse phoned the patients at least 6 months after stroke onset and invited the patients to the outpatient clinic for investigations. Due to practical reasons, investigations were only possible during a certain period of the study.

Risk factors including hypertension, smoking, diabetes mellitus, myocardial infarction, angina pectoris, peripheral artery disease, atrial fibrillation and previous admission for depression were registered on admittance. Patients were asked about a prior history of depression. Previous admission for depression was defined as depression requiring use of selective serotonin reuptake inhibitor treatment at any time before stroke onset. Hypertension was defined as prior use of antihypertensive medication. Current smoking was defined as smoking at least 1 cigarette/day. Diabetes mellitus was considered present if the patient was on glucose-lowering diet or medication. Angina pectoris, myocardial infarction and peripheral artery disease were considered present if diagnosed by a physician any time before stroke onset. Atrial fibrillation required ECG confirmation any time prior to stroke onset or during the hospital stay. A history of prior stroke was registered. The etiology of ischemic stroke was determined by TOAST (the Trial of Org 10172 in Acute Stroke Treatment classification). Leukoaraiosis was defined as the presence of hypodense periventricular abnormalities in the deep white matter on MRI. Isolated acute ischemic lesions on DWI were defined as lacunar infarctions if $<1.5 \mathrm{~cm}$ and located subcortical or in the brainstem [19]. All other acute ischemic lesions were defined as embolic infarctions. Embolic infarctions comprised subcortical and brainstem infarction $\geq 1.5 \mathrm{~cm}$, cortical infarction, mixed cortical and subcortical infarction and cerebellar infarction. The National Institutes of Health Stroke Scale was used to assess stroke severity on admission. The mRS score was assessed on day 7 or at discharge, if discharged earlier.

\section{Tests}

The MMSE was administered according to standard instructions [16]. This test consists of 30 different items testing specified areas of mental functioning on a pass-or-fail basis. Each person achieved a score between 0 and 30. Age-matched normal control data were obtained from a large population-based normative study [20].

In the Hopkins Verbal Leaning Test-Revised (HVLT-R) [21], verbal learning and recall, which are associated with everyday functioning in the elderly, are tested [22]. The HVLT-R is sensitive to early changes both in MCI $[23,24]$ and stroke $[25,26]$. It consists of a list of 12 words read aloud 3 times. The 12 words belong to 3 different semantic categories with 4 words in each category. After each presentation, the person tested was asked to repeat as many words from the list as possible. The number of words remembered on each trial is given, as well as the sum of words remembered across trials 1-3. Twenty minutes after this part of the test, the person was asked to say as many words from the list as possible without any further cues (delayed recall). The total number of words remembered in the delayed recall condition is given, as well as the percentage of words remembered in delayed recall relative to the best result from a single trial in the original presentation (percent retained). After the free recall condition, a total of 24 words, including the words from the original list, were read aloud, and the person was asked to identify the words from the list. The total number of words correctly identified (true positive) and the total number of words falsely 
identified as belonging to the list (false positive) are given, as well as the ratio of true-positive results to the total number of words identified as belonging to the list (discrimination index). Normal control data are derived from a study of healthy adults between 60 and 85 years of age [27].

The Stroop test [28] is one of the most frequently used tests of executive function [29], and it has been used in many studies of stroke patients [30-32]. Three cards were presented, each containing 5 columns of 20 sets of symbols, and the task was to read as many symbols as possible in $45 \mathrm{~s}$. In the subtest word reading, the symbols are the color names blue, green and red printed in black ink. In the subtest color reading, the symbols are XXXX printed in blue, green or red colors. In the subtest color-word reading, the symbols are color names printed in contrasting colors (e.g. the word red printed in green color), and the task is to read the color of the words (not the word itself). For each subtest, the score was the number of symbols read in $45 \mathrm{~s}$. In addition, to further evaluate the specific effect of color-word interference without regard to reading speed, an interference score was calculated according to the formula

$$
\text { interference }=\text { color-word }-[(\text { word } \times \text { color }) /(\text { word }+ \text { color })] \text {. }
$$

Norms used for this test are from the control group of a study comparing patients with AD with 36 healthy elderly (mean age 72.9 years, $S D=8.3$ ) [12].

For each variable, differences in mean results between the patient group and the normative group were tested for statistical significance using the $t$ test for independent groups. Similar statistics were calculated to test differences between selected subgroups. All statistics were calculated on STATA 11 (StataCorp LP, College Station, Tex., USA).

\section{Results}

Fifty patients were included in the present study, as planned. During the study period, MRI was performed in $286(73.5 \%)$ patients with cerebral infarction and mRS $\leq 2$ after 1 week (age $>60$ years). In total, 125 patients meeting the inclusion criteria were not included in the present study due to the limited follow-up period. Very few patients who were invited for participation declined. Table 1 shows demographic results of the 50 study patients and the 125 patients not included. The study patients were somewhat younger and had somewhat a better functional score after 1 week. The frequency of leukoaraiosis was lower among the study patients. Otherwise there were no difference between study patients and the other patients. Median time to neuropsychological investigation was 381 days (interquartile range 320-530 days).

Anatomical locations of the infarctions were: 13 lacunar infarctions in basal ganglia/ internal capsula, 7 subcortical infarctions in the MCA territory, 15 cortical infarctions in the MCA territory, 8 mixed cortical and subcortical infarctions in the MCA territory, 2 infarctions in the thalamus, 2 cortical infarctions in the occipital lobe, 2 cerebellar infarctions and 1 infarction in the brainstem.

The main findings are presented in tables 2-4. In the MMSE, a significant difference between the patients and the normal controls was found in the group aged 60-64 years. Other comparisons were not significant. In the HVLT-R, significant differences between the patients and the controls were found in all measures, except percent retained words in delayed recall and number of false-positive responses in the recognition test. In the Stroop test, significant differences between the patients and the controls were found in all measures of reading speed, but not in interference. 
Table 1. Demographic data of the study patients and comparable patients with cerebral infarction

\begin{tabular}{lccl}
\hline & $\begin{array}{l}\text { Study group } \\
(\mathrm{n}=50)\end{array}$ & $\begin{array}{l}\text { Excluded patients } \\
(\mathrm{n}=125)\end{array}$ & $\mathrm{p}$ \\
\hline Mean age, years & 70.2 & 73.6 & 0.01 \\
Males & $32(64 \%)$ & $71(57 \%)$ & 0.20 \\
Married & $36(75 \%)$ & $76(63.3 \%)$ & 0.82 \\
Employed & $9(19.2 \%)$ & $20(17.4 \%)$ & 0.32 \\
History & $8(16.7 \%)$ & $25(20.7 \%)$ & 0.73 \\
$\quad$ Daily smoking & $28(56 \%)$ & $75(60 \%)$ & 1.00 \\
$\quad$ Hypertension & $7(14 \%)$ & $19(15.5 \%)$ & 0.17 \\
$\quad$ Diabetes mellitus & $8(16 \%)$ & $33(26.4 \%)$ & 0.78 \\
$\quad$ Atrial fibrillation & $4(8.2 \%)$ & $14(11.2 \%)$ & 0.036 \\
$\quad$ Prior cerebral infarction & $3(6 \%)$ & $24(19.2 \%)$ & 0.81 \\
$\quad$ Myocardial infarction & $6(12 \%)$ & $18(14.4 \%)$ & 0.63 \\
$\quad$ Angina pectoris & $6(13 \%)$ & $17(16.8 \%)$ & 0.55 \\
$\quad$ Prior depression & & 3.1 & 0.009 \\
Mean scores & 2.7 & 1.2 & 0.88 \\
$\quad$ NIHSS score on admission & 0.9 & $24(19.4 \%)$ & \\
mRS score after 1 week & $11(22 \%)$ & $30(24.2 \%)$ & \\
TOAST & $9(18 \%)$ & $22(17.7 \%)$ & \\
$\quad$ Atherosclerosis & $10(20 \%)$ & $1(0.8 \%)$ & \\
Cardiac embolism & $0(0 \%)$ & $47(37.9 \%)$ & \\
Small-vessel disease & $20(40 \%)$ & $89(71.2 \%)$ & 0.86 \\
Other & & $36(28.8 \%)$ & \\
$\quad$ Unknown & $35(70 \%)$ & $77(62.1 \%)$ & 0.007 \\
MRI & $15(30 \%)$ & & \\
$\quad$ Embolic infarction & $19(38.9 \%)$ & & \\
Lacunar infarction & & & \\
Leukoaraiosis & & & \\
\hline
\end{tabular}

Numbers of patients are shown unless otherwise indicated.

Table 2. MMSE results of patients with mild stroke compared to normative data in controls (Crum et al. [20])

\begin{tabular}{llrlll}
\hline \multirow{2}{*}{ Age group } & & $\mathrm{n}$ & \multicolumn{2}{l}{ MMSE } & \multirow{2}{*}{ p value } \\
\cline { 5 - 5 } & & & mean & SD & \\
\hline \multirow{2}{*}{$60-64$ years } & patients & 16 & 26.9 & 1.4 & 0.026 \\
& controls & 1,294 & 28 & 2.0 & \\
$65-69$ years & patients & 11 & 26.6 & 1.7 & 0.41 \\
& controls & 1,931 & 27 & 1.6 & \multirow{2}{*}{0.38} \\
$70-74$ years & patients & 7 & 27.6 & 1.7 & \multirow{2}{*}{0.13} \\
$75-79$ years & controls & 1,477 & 27 & 2.8 & \\
& patients & 13 & 26.9 & 2.3 & \\
\hline
\end{tabular}


Table 3. HVLT-R results in patients with mild stroke compared to normative data in controls (Vanderploeg et al. [27])

\begin{tabular}{lcccccc}
\hline \multirow{2}{*}{ HVLT measure } & \multicolumn{2}{c}{ Patients $(\mathrm{n}=49)$} & & \multicolumn{2}{c}{ Controls $(\mathrm{n}=394)$} & \multirow{2}{*}{ p value } \\
\cline { 2 - 3 } & mean & SD & & mean & SD & \\
\hline Trial 1 & 4.2 & 1.9 & & 4.8 & 1.7 & 0.022 \\
Trial 2 & 6.0 & 1.8 & & 7.4 & 2.1 & $<0.001$ \\
Trial 3 & 6.9 & 2.2 & & 8.4 & 2.2 & $<0.001$ \\
Sum of trials 1-3 & 16.9 & 5.1 & & 20.6 & 5.2 & $<0.001$ \\
Delayed recall & 5.9 & 2.6 & & 7.8 & 2.7 & $<0.001$ \\
Percent retained & 87.4 & 33.6 & & 89.0 & 25.8 & 0.69 \\
Recognition & & & & & & \\
$\quad$ True positive & 10.1 & 1.6 & & 11.3 & 1.0 & $<0.001$ \\
$\quad$ False positive & 1.5 & 1.9 & & 1.6 & 1.5 & 0.67 \\
Discrimination index & 8.7 & 1.9 & & 9.7 & 1.9 & 0.001 \\
\hline
\end{tabular}

Table 4. Stroop color-word interference test results in patients with mild stroke compared to normative data in controls (Fisher et al. [12])

\begin{tabular}{llllllll}
\hline Stroop measure & \multicolumn{2}{l}{ Patients $(\mathrm{n}=49)$} & & \multicolumn{2}{c}{ Controls $(\mathrm{n}=36)$} & \multirow{2}{*}{$\mathrm{p}$ value } \\
\cline { 2 - 3 } & mean & $\mathrm{SD}$ & & mean & $\mathrm{SD}$ & \\
\hline Word reading & 79.2 & 14.4 & & 96.6 & 15.8 & $<0.001$ \\
Color reading & 47.0 & 13.5 & & 64.9 & 13.9 & $<0.001$ \\
Color-word reading & 23.6 & 11.5 & & 33.4 & 10.8 & $<0.001$ \\
Interference & -5.5 & 8.2 & & -5.2 & 8.6 & 0.87 \\
\hline
\end{tabular}

In addition to the main comparisons between the whole group and normative data, several subgroup analyses were carried out. There were no significant differences between patients with lacunar or embolic stroke. Comparing patients with or without leukoaraiosis on MRI, only one significant difference was found (better performance of the non-leukoaraiosis group on the Stroop color reading; $\mathrm{p}=0.05$ ). Patients with predominantly left hemisphere infarction $(n=24)$ did significantly worse on the MMSE than those with predominantly right hemisphere infarction $(\mathrm{n}=19 ; \mathrm{p}=0.005)$. They did, however, significantly better on HVLT-R trial $1(\mathrm{p}=0.03)$, trial $2(\mathrm{p}=0.02)$ and discrimination index $(\mathrm{p}=0.006)$, and Stroop word reading $(\mathrm{p}=0.04)$. Otherwise no significant differences between these groups were found.

\section{Discussion}

The main findings of this study were that elderly patients with mild stroke had significant difficulties both in memory and executive functions. Moreover, distinct cognitive features emerged. Patients showed deficient verbal learning and recall, but no specific deficit in delayed free recall. Reading speed was reduced at all difficulty levels, but no specific deficit in color-word interference was found. The MMSE showed weak sensitivity in detecting cognitive deficits in elderly patients with mild stroke, particularly in those aged $>65$ years. This 
may reflect that the cognitive dysfunction seen in these patients is mild to moderate, and that the MMSE is better suited to detect severer brain dysfunction.

The relatively intact verbal free recall in this patient group suggests that patients with mild stroke show a different pattern of memory dysfunction from that normally seen in patients with early $\mathrm{AD}$ and amnesic $\mathrm{MCI}$, and also in other diseases involving medial temporal lobe structures, such as mesial temporal lobe epilepsy [33]. Thus, this finding is consistent with a relatively normal functioning of medial temporal lobe structures in mild stroke patients.

The somewhat unexpected lack of interference deficit in the Stroop test is harder to explain in terms of brain anatomy. Recent reviews $[34,35]$ point out that the interference effect is difficult to localize in lesion studies, but at least one large study [36] indicates that lesions involving superior medial frontal regions affect interference. Imaging studies have more consistently suggested a possible crucial role of medial frontal lobe structures, particularly of the anterior cingulate cortex, for the interference effect in the Stroop test. Thus, the finding of a normal interference score in patients with mild stroke may be taken as an indication of intact functioning of the medial frontal lobe. Evidence for the anatomical localization of the interference effect is relatively weak, and our data do not permit any firm conclusions about localization. However, infarctions are more common in lateral brain areas (subserved by the middle cerebral artery), and infarctions in medial frontal lobe structures (subserved by the anterior cerebral artery) are relatively rare [37].

Taken together, the profile of cognitive deficits in mild stroke is compatible with a dysfunction in the cerebral hemispheres with relative sparing of midline structures. Subgroup analysis did not reveal consistently significant effects of leukoaraiosis or type of lesion (embolic or lacunar infarction). A possible effect of lateralization is seen in some tasks. Patients with predominantly left-sided lesions did worse on the MMSE, as expected from earlier studies [38]. However, better results on measures of verbal recall, verbal recognition memory and word reading are not anticipated in patients with left-sided lesions. Tests with a verbal content are generally expected to be more affected by lesions of the left hemisphere [39]. One explanation may be that patients with small left-sided infarctions are more likely to be hospitalized than patients with right-sided infarctions of similar magnitude, leading to a selection bias of the patient population [40]. This could also explain why the difference in MMSE results is in the expected direction. In more sensitive tests, the selection bias may affect the results, but because the MMSE is generally less sensitive, it is less likely to be affected by a selection bias in the patients with the smallest lesions. Thus, the difference in the MMSE may primarily reflect a difference in the patients with larger lesions and more pronounced cognitive deficits.

The patients included in this study were not entirely representative of the whole population of elderly with mild stroke (table 1). They appear to be among the better-functioning patients in this group. This is not necessarily a problem for the conclusions, because even in this group clear deviations from normal controls are seen. The main methodological problem in this study is the lack of a specified control group of healthy individuals. The normative data used are from English-speaking populations, and translation problems and crosscultural differences may have affected the results. Another problem is that each test has a different control group. This makes comparisons across tests difficult, and caution must be exercised in the interpretation of results. We did not assess depression, and thus a possible effect of mild depression on the results cannot be excluded.

However, the main findings seem to be fairly robust, with statistically significant differences in the variables showing deficits, and a similar lack of significance on the variables where deficits were not shown. Also, the findings are in agreement with the results of other studies. However, further studies in cohorts including matched control groups are clearly needed to confirm the cognitive profile of elderly patients with mild stroke and the main 
conclusions drawn in our study. To further explore the possible applicability of the cognitive profile in differential diagnosis, comparisons with matched groups of patients with early AD and/or amnesic MCI should be done. Another suggestion for further studies is to expand the test battery applied. In particular, comprehensive testing of executive functions probably demands a wider test range. Our conclusions apply to a limited range of cognitive functioning, and a more comprehensive range of tests may uncover other aspects of the cognitive profile in mild stroke.

\section{Acknowledgments}

We thank Liv Sissel Dale and Maria Reed Grimeland for their assistance in the data collection.

\section{References}

1 Barker-Collo S, Feigin V: The impact of neuropsychological deficits on functional stroke outcome. Neuropsychol Rev 2006;16:53-64.

-2 Donovan NJ, Kendall DI, Heaton SC, Kwon S, Velozo CA, Duncan PW: Conceptualizing functional cognition in stroke. Neurorehabil Neural Repair 2008;22:122-135.

3 Erkinjuntti T, Gauthier S: The concept of vascular cognitive impairment; in Giannakopoulus P, Hof PR (eds): Dementia in Clinical Practice. Front Neurol Neurosci. Basel, Karger, 2009, vol 24, pp 79-85.

-4 deHaan EH, Nys GM, Van Zandvoort MJ: Cognitive function following stroke and vascular cognitive impairment. Curr Opin Neurol 2006;19:559-564.

-5 Snaphaan L, de Leeuw FE: Poststroke memory function in nondemented patients. Stroke 2006;37: 198-203.

-6 Mansueti L, de Frias CM, Bub D, Dixon RA: Exploring cognitive effects of self reported mild stroke in older adults: selective but robust effects on story memory. Neuropsychol Dev Cogn B Aging Neuropsychol Cogn 2008;15:545-573.

-7 Schouten EA, Schiemanck SK, Brand N, Post MWM: Long-term deficits in episodic memory after ischemic stroke: evaluation and prediction of verbal and visual memory performance based on lesion characteristics. J Stroke Cerebrovasc Dis 2009;18:128-138.

-8 Desmond DW: The neuropsychology of vascular cognitive impairment: is there a specific cognitive deficit? J Neurol Sci 2004;226:3-7.

-9 Braak H, Braak E: Neuropathological staging of Alzheimer-related changes. Acta Neuropathol 1991; $82: 239-259$.

10 Salmon DP: Disorders of memory in Alzheimer's disease; in Cermak LS (ed): Handbook of Neuropsychology, ed 2; vol 2: Memory and Its Disorders. Amsterdam, Elsevier, 2000, pp 155-195.

-11 Salmon DP, Bondi MW: Neuropsychological assessment of dementia. Annu Rev Psychol 2009;60: 257-282.

12 Fisher M, Freed DM, Corkin S: Stroop Color-Word Test performance in patients with Alzheimer's disease. J Clin Exp Neuropsychol 1990;12:745-758.

-13 Markesbery WR: Neuropathological alterations in mild cognitive impairment: a review. J Alzheimers Dis 2010;19:221-228.

-14 Belleville S, Sylvain-Roy S, de Boysson C, Mènard MC: Characterizing the memory changes in persons with mild cognitive impairment. Prog Brain Res 2008;169:365-375.

-15 Pike KE, Savage G: Memory profiling in mild cognitive impairment: can we determine risk for Alzheimer's disease? J Neuropsychol 2008;2:361-372.

-16 Folstein MF, Folstein SE, McHugh PR: 'Mini-Mental State'. A practical method for grading the cognitive state of patients for the clinician. J Psychiatr Res 1975;12:189-198.

-17 Pendlebury ST, Cuthbertson FC, Welch SJV, Mehta Z, Rothwell PM: Underestimation of cognitive impairment by Mini-Mental State Examination versus the Montreal Cognitive Assessment in patients with transient ischemic attack and stroke: a population-based study. Stroke 2010;41:1290-1293. 
18 Bour A, Rasquin S, Boreas A, Limburg M, Verhey F: How predictive is the MMSE for cognitive performance after stroke? J Neurol 2010;257:630-637.

-19 Wessels T, Wessels C, Ellsiepen A, Reuter I, Trittmacher S, Stolz E, Jauss M: Contribution of diffusion-weighted imaging in determination of stroke etiology. Am J Neuroradiol 2006;27:35-39.

-20 Crum RM, Anthony JC, Bassett SS, Folstein MF: Population-based norms for the Mini-Mental State Examination by age and education level. JAMA 1993;269:2386-2391.

-21 Benedict RHB, Schretlen D, Groninger L, Brandt J: The Hopkins Verbal Learning Test-Revised: normative data and analysis of inter-form and test-retest reliability. Clin Neuropsychol 1998;12:43-55.

-22 Gross AL, Rebok GW, Unverzagt FW, Willis SL, Brandt J: Word list memory predicts everyday function and problem-solving in the elderly: results from the ACTIVE cognitive intervention trial. Neuropsychol Dev Cogn B Aging Neuropsychol Cogn 2011;18:129-146.

-23 Tremont G, Miele A, Smith MM, Westervelt HJ: Comparison of verbal memory impairment rates in mild cognitive impairment. J Clin Exp Neuropsychol 2010;32:630-636.

-24 de Jaeger CA, Schrijnemaekers MC, Honey TM, Budge MM: Detection of MCI in the clinic: evaluation of the sensitivity and specificity of a computerized test battery, the Hopkins Verbal Learning Test and the MMSE. Age Ageing 2009;38:455-460.

-25 Stewart KJ, Gale SD, Diamond PT: Early assessment of post-stroke patients entering acute inpatient rehabilitation. Utility of the WASI and HVLT-R. Am J Phys Med Rehabil 2002;81:223-228.

-26 Skidmore ER, Whyte EM, Holm MB, Becker JT, Butters MA, Dew MA, Munin MC, Lenze EJ: Cognitive and affective predictors of rehabilitation participation after stroke. Arch Phys Med Rehabil 2010;91:203-207.

-27 Vanderploeg RD, Schinka JA, Jones T, Small BJ, Graves AB, Mortimer JA: Elderly norms for the Hopkins Verbal Learning Test-Revised. Clin Neuropsychol 2000;14:318-324.

28 Golden C: Stroop Color and Word Test. Manual. Chicago, Stoelting, 1978.

-29 Jurado MB, Rosselli M: The elusive nature of executive functions: a review of our current understanding. Neuropsychol Rev 2007;17:213-233.

- 30 Verdelho A, Madureira S, Ferro JM, Basile AM, Chabriat H, Erkinjuntti T, Fazekas F, et al: Differential impact of cerebral white matter changes, diabetes, hypertension and stroke on cognitive performance among non-disabled elderly. The LADIS study. J Neurol Neurosurg Psychiatry 2007;78:13251330.

- 31 Koga H, Takashima Y, Murakawa R, Uchino A, Yuzuriha T, Yao H: Cognitive consequences of multiple lacunes and leukoaraiosis as vascular cognitive impairment in community-dwelling elderly individuals. J Stroke Cerebrovasc Dis 2009;18:32-37.

-32 Tang WK, Chen Y, Lam WWM, Mok V, Wong A, Ungvari GS, Xiang YT, Wong KAS: Emotional incontinence and executive function in ischemic stroke: a case-controlled study. J Int Neuropsychol Soc 2009; 15:62-68.

-33 Saling MM: Verbal memory in mesial temporal lobe epilepsy: beyond material specificity. Brain 2009;132:570-582.

-34 Goldberg E, Bougakov D: Neuropsychologic assessment of frontal lobe dysfunction. Psychiatr Clin North Am 2005;28:567-580.

-35 Alvarez JA, Emory E: Executive function and the frontal lobes: a meta-analytic review. Neuropsychol Rev 2006; 16:17-42.

- 36 Stuss DT, Floden D, Alexander MP, Levine B, Katz D: Stroop performance in focal lesion patients: dissociation of processes and frontal lobe lesion location. Neuropsychologia 2001;39:771-786.

- 37 Kumral E, Bayulkem G, Evyapan D, Yunten N: Spectrum of anterior cerebral artery territory infarction: clinical and MRI findings. Eur J Neurol 2002;9:615-624.

- 38 Tombaugh TN, McIntyre NJ: The Mini-Mental State Examination: a comprehensive review. J Am Geriatr Soc 1992;40:922-935.

- 39 Hugdahl K: Lateralization of cognitive processes in the brain. Acta Psychol 2000;105:211-235.

-40 Naess H, Waje-Andreassen U, Thomassen L, Myhr KM: High incidence of infarction in the left cerebral hemisphere among young adults. J Stroke Cerebrovasc Dis 2006;15:241-244. 